\title{
Secondary Prevention of Cancer in the Older Individual
}

\section{María Vallet-Regi ${ }^{1,2 *}$, Miguel Manzano ${ }^{1,2}$, Leocadio Rodriguez-Mañas ${ }^{3}$, Manuel Ramírez Orellana ${ }^{4}$, Alfredo Carrato ${ }^{5}$, Pablo L Ortiz Romero ${ }^{6}$ and Lodovico Balducci ${ }^{7}$}

\author{
${ }^{1}$ Departamento de Química Inorgánica y Bioinorgánica, Universidad Complutense de Madrid, Spain \\ ${ }^{2}$ Networking Research Center on Bioengineering, Biomaterials and Nanomedicine (CIBER-BBN), Spain \\ ${ }^{3}$ Service of Geriatrics, Hosptal Universitario de Getafe, Spain \\ ${ }^{4}$ Departament of Hematology and Pedriatic Oncology, Hospital Infantil Universitario Niño Jesús, Spain \\ ${ }^{5}$ Ramón y Cajal University Hospital, Alcalá de Henares University, Spain \\ ${ }^{6}$ Dermatology Department, Hospital Universitario, Spain \\ ${ }^{7} \mathrm{H}$ Lee Moffitt Cancer Center \& Research Institute, University of South Florida, USA
}

\begin{abstract}
The incidence and the mortality of cancer increase with age. This article explores the possibility of decreasing cancerrelated mortality in the aged with secondary prevention of cancer deaths that entails early diagnosis of cancer through the screening of asymptomatic older individuals.

We establish that screening of asymptomatic individuals should be based on physiologic rather than chronologic age that may be estimated from a comprehensive geriatric assessment and possibly with the utilization of biologic markers of aging.

It is reasonable to offer some form of screening for lung and colorectal cancer to individuals with a life expectancy of at least five years and screening for breast and prostate cancer to women and men respectively with a life expectancy of at least ten years. The ideal number of screening sessions and the ideal interval between screening sessions is unestablished.

The aging of the population, the diversity of the older population, the development of new and more sensitive screening interventions, the discovery of new biologic markers of cancer and age represent the main challenges in studying the value of cancer screening in the aged. Probably the most reliable information may be obtained from rapid-learning databases in which information related to each person's physiologic age is included.
\end{abstract}

\section{Introduction}

Worldwide, the incidence and prevalence of cancer increase with age [1]. In the meantime the risk of cancerrelated mortality increases with age at diagnosis [2-4]. As the world population is aging, cancer in the older person is an ever more common problem, and the reduction of cancer deaths in older individuals represents the most urgent goal of cancer control. In this article we explore secondary cancer prevention as representing a strategy to reduce the risk of mortality in the aged. This hypothesis is based on four considerations:

i. Several studies showed that the practice of screening asymptomatic individuals for cancer becomes less common with the aging of the population [5].

ii. The average life expectancy of the Western population is rapidly increasing1. Consequently the benefits of early detection of cancer that emerges several years after diagnosis may be present even for those undergoing screening at an advanced age.

iii. New forms of cancer treatment, including minimally invasive surgery [6], stereotactic radio surgery [7], and

\begin{abstract}
*Corresponding author: Maria Vallet-Redgi, Facultad de Farmacia, Departamento de Química Inorgánica y Bioinorgánica, Universidad Complutense de Madrid, Instituto de Investigación Sanitaria Hospital 12 de Octubre i+12, Networking Research Center on Bioengineering, Biomaterials and Nanomedicine (CIBER-BBN), Madrid, Spain, Tel: +34913941861, E-mail: vallet@ucm.es
\end{abstract}

Received: November 21, 2016; Accepted: March 17, 2017; Published online: March 20, 2017

Citation: Vallet-Regi M, Manzano M, Rodriguez-Mañas L, et al. (2017) Secondary Prevention of Cancer in the Older Individual. Trends Geriatr Healthc 1(1):14-22

Copyright: (C) 2017 Vallet-Regi M, et al. This is an open-access article distributed under the terms of the Creative Commons Attribution License, which permits unrestricted use, distribution, and reproduction in any medium, provided the original author and source are credited. 
targeted systemic therapy [8], are associated with decreased risk of complications. They may be safely utilized in individuals with limited tolerance of stress who might be hurt by more aggressive treatments.

It is now possible to personalize cancer screening according to individual life expectancy and tolerance of stress, as the estimate of a person's physiologic age is becoming more precise $[1,9]$.

After reviewing the principles and the effectiveness of cancer screening and early detection, this article will explore the benefits and risks of secondary cancer prevention in older individuals. In particular we will review the current evidence and the limitations of previous clinical trials. At the end we will propose a conceptual framework to guide the screening of older individuals for cancer, and we will propose a research agenda.

\section{Principles of Cancer Screening and Early De- tection}

In this section we will examine the assumptions that justify secondary cancer prevention, the endpoints of clinical trials, the ideal characteristics of the screening test and the potential advantages and disadvantages of screening asymptomatic older individuals for cancer [10].

\section{Assumptions that justify secondary cancer pre- vention}

Three assumptions underlie the employment of cancer screening and early detection:

i. There is an early, asymptomatic stage when cancer may be diagnosed with screening.

ii. Treatment of cancer at an early, asymptomatic stage is associated with improved chances of cure.

iii. The risks of the treatment are minimal when compared with the potential benefits.

Cost considerations may be involved in the decision to implement cancer screening as a public health policy. Budget restrictions compel governments to prioritize the policies that will produce the best return for the lowest investment. By necessity, this determination is arbitrary. In the USA a policy is considered cost effective when the cost per year of life saved is equivalent to or lower than the cost of one year of life with kidney dialysis [11].

\section{End point of clinical trials and related questions}

There is consensus that the only acceptable end point of clinical trials of secondary cancer prevention is a reduction of the cancer-related mortality in the screened population. Intermediate end points proposed in the past have proven flawed and should not be entertained [10] (Table 1).
Table 1: Intermediate end points of clinical trials and potential biases.

\begin{tabular}{|l|l|}
\hline Intermediate end point & Potential bias \\
\hline $\begin{array}{l}\text { Improved cancer-related } \\
\text { survival in the screened } \\
\text { population }\end{array}$ & $\begin{array}{l}\text { Lead time bias: In reality the } \\
\text { survival of the screened population } \\
\text { has not been prolonged; only the } \\
\text { duration of time during which they } \\
\text { have been known to have cancer } \\
\text { has been prolonged }\end{array}$ \\
\hline $\begin{array}{l}\text { Screening lead to } \\
\text { diagnosis of cancer at an angth time bias: The cancers } \\
\text { early stage }\end{array}$ & $\begin{array}{l}\text { Lenges } \\
\text { diagnosed through screening are } \\
\text { less aggressive than those diag- } \\
\text { nosed when the patient is symp- } \\
\text { tomatic. In other words, some of } \\
\text { the most aggressive cancers es- } \\
\text { cape screening, as they emerge } \\
\text { in the interval during the screen- } \\
\text { ing sessions }\end{array}$ \\
\hline $\begin{array}{l}\text { Over-Detection bias: most of } \\
\text { diagnosis of more cancer } \\
\text { the cancer diagnosed through } \\
\text { screening may not become clin- } \\
\text { ically relevant during a person's } \\
\text { lifetime. This type of bias is of par- } \\
\text { ticular concern to older individuals } \\
\text { with limited life expectancy. }\end{array}$ \\
\hline
\end{tabular}

When dealing with older individuals, it is legitimate to ask if alternative endpoints should be considered, such as prolongation of overall survival, prolongation of active life expectancy, and improvement of quality of life [12]. With the aging of the population, cancer becomes a major cause of mortality, and it is reasonable to expect that a reduction in cancer deaths may be associated with improved overall survival. In the meantime it is important to establish that the price of reduced mortality does not include functional dependence and compromised quality of life.

\section{The ideal screening test}

It is self-evident that a screening test should be minimally invasive and reasonably priced [10]. A new problem emerged in an era of precision technology: screening tests may lead to unnecessary interventions that may increase the risk and the cost of screening. The cause of this problem is twofold. First, some new tests are so sensitive that they detect an excess of cancer that may never become clinically relevant. For example, the diagnostic yield of breast MRI or three-dimensional mammography is superior to that of conventional or digital mammography without proof that the newest tests have affected the cancer-related mortality. Second, some tests discover lesions that are so early they may never become cancerous. For example, about $80 \%$ of young sexually active women may test positive for HPV1, which is the most of common cause of cervical cancer [13]. Only a small minority of these infections will eventually cause cancer, and this finding should not lead to a number of unnecessary hysterectomies. 
Citation: Vallet-Regi M, Manzano M, Rodriguez-Mañas L, et al. (2017) Secondary Prevention of Cancer in the Older Individual. Trends Geriatr Healthc 1(1):14-22

Table 2: Advantages and disadvantages of cancer screening in the aged.

\section{Advantages}

- Increased prevalence of cancer $\rightarrow$ improved predictive value positive of screening tests

- Early diagnosis $\rightarrow$ reduced risk of complications from emergency surgery

- Increased cancer related mortality with age $\rightarrow$ possibility to improve overall survival

\section{Disadvantages}

- Reduced life expectancy $\rightarrow$ reduced survival benefits and risk of over-detection

- Decreased tolerance of stress $\rightarrow$ increased risk of diagnosis and treatment-related complications

- Decreased yield of cancer as a result of previous screening

Table 3: Benefits of cancer screening from randomized clinical trials.

\begin{tabular}{|l|l|l|l|l|}
\hline Cancer & Screening test & Age range & $\begin{array}{l}\text { Reduction in cancer } \\
\text { related deaths }\end{array}$ & $\begin{array}{l}\text { Improvement in } \\
\text { overall survival }\end{array}$ \\
\hline Breast & Mammography & $50-69$ & Yes & No \\
\hline Large bowel & $\begin{array}{l}\text { Fecal Occult Blood (FOB) } \\
\text { Flexible sygmoidoscopy }\end{array}$ & $50-80$ & Yes & No \\
\hline Lung & Low-dose CT scan & $55-75$ & Yes & Yes \\
\hline Prostate & PSA & $50-70$ & Yes & No \\
\hline Liver (hepatocellular) & $\begin{array}{l}\text { Circulating Alfa Fetoprotein (AFP) } \\
\text { Liver US }\end{array}$ & $40-70$ & Yes & No \\
\hline
\end{tabular}

Possible benefits and risks of screening older individuals for cancer

Table 2 describes how age may affect cancer screening [10]. As the prevalence of cancer increases in the population, the specificity and the predictive value positive of screening tests improve as well, with reduction in the risk of false positive tests. Also, early detection of cancer may lead to increased use of minimally invasive surgery and lessen the need for emergency surgery, whose serious complications, including death, increase with the patient's age.

In the meantime the increased risk of competitive causes of death and of treatment complications may reduce the benefits of screening. For this reason, we advocate in this article a policy of screening older individuals according to their physiologic age-that is, life expectancy and tolerance of stress-rather than chronologic age. Also, it is not clear how beneficial multiple serial screening sections may be, when most prevalence cases have been detected with the initial ones.

\section{Current Evidence of the Benefits of Screening Older Individuals with Cancer}

Table 3 summarizes the evidence from randomized clinical trials that cancer screening is beneficial.

\section{Breast cancer}

A systematic review of 8 randomized controlled studies [14] indicates that a $15 \%$ approximate reduction in mortality risk may be obtained by screening asymptomatic women for breast cancer ages 50-69 with serial mammograms. It is not clear how much the reduction in mortality was due to screening or to simultaneous improvement in cancer management including the use of adjuvant therapy. The same conclusions were reached by the U.S. Preventive Service Task Force [15], which issued a recommendation to screen average-risk women with biennial mammograms from ages 50-75. It is worthy of mention that mammography failed to effect a reduction in breast cancer-related mortality over 20 years of follow up in women ages 50-60 in the large Canadian study[16]. This study, which was highly publicized, compared physical examination of the breast by a professional and a single-view screening mammography. This mammographic technique is now obsolete.

Circumstantial evidence suggests that screening mammography may be beneficial to older women. A retrospective review of the Medicare-matched Surveillance Epidemiology and End Results (SEER) data demonstrated that serial mammograms reduced the risk of breast cancer-related mortality up to age 85 [17]. In a case-controlled study, mammography up to age 80 was associated with a mortality reduction [18]. In a prospective study of breast cancer survivors with an age range of 65-85 years, each mammogram was associated with a 0.69 fold decrease in the risk of breast cancer mortality [19]. In the meantime, in Vermont an inverse relation was found between the use of screening mammography and breast cancer deaths up to age 85 [20]. In a review of 7 studies exploring benefits and risks of mammography in women 65 and older, older women non affected by severe co morbidity appeared to experience an improved survival from serial mammography [21].

A number of questions remain unanswered including the role of new imaging techniques (breast MRI, 3D breast tomography), the ideal interval between screening sessions, and the value of clinical examination of the breast by a health-care professional. 
Limited evidence indicates that some form of breast cancer screening may reduce mortality and morbidity of breast cancer in women with a life expectancy of at least 5 years. As partial and even total mastectomy now can be performed with local anesthesia, the contraindications to breast surgery are becoming rarer and rarer, even for women of advanced age.

\section{Colorectal cancer}

Asymptomatic individuals may be screened for colorectal cancer with several methods [22] that include:

i. Serial fecal examinations for occult blood with guaiac (FOB) or Fecal Immunochemical Test (FIT)

\section{ii. Serial fecal examination for DNA}

iii. Serial endoscopies, which include sigmoidoscopies and colonoscopies

iv. Computed Tomographic Colonography (CTC).

According to five randomized controlled studies, serial sygmoidoscopy reduced the colorectal cancer mortality by $27 \%$ by $10-11$ years of follow up; and in five randomized controlled studies, serial determinations of FOB reduced it by $9-22 \%$ over a period of 9-30 years. Despite the absence of randomized controlled studies, serial colonoscopies are commonly used, because they allow the detection and resection of adenomatous polyps, which are precancerous lesions, in addition to visualizing the whole large bowel [23]. The exact role of FIT and stool DNA in the detection of colorectal cancer is undefined. Clearly both tests are more specific than FOB, and stool DNA is more sensitive but less specific than FIT.

Several studies explored the sensitivity of CTC in detecting colonic adenomas. Using colonoscopy as the gold standard, the sensitivity of CTC varied with the size of the tumor and ranged from $66 \%$ to $97 \%$. CTC requires the same bowel preparation as colonoscopy and involves the risk of a small dose of radiation, but the risk of perforation is lower with CTC. It is unclear in which patients CTC may be more convenient and safer than colonoscopy.

The USPSTF recommends screening individuals ages 50-75 for colorectal cancer [22]. Most randomized clinical trials included individuals only up to age 80 , however. We believe that life expectancy and tolerance of treatment should determine the decision of screening older individuals, more so than chronologic age. Serial determinations of FOB every 1-2 years, serial sigmoidoscopies every 3-5 years and serial colonoscopies every 10 years appear to be acceptable strategies. Most likely the colonoscopy is the most practical and convenient. As the risk of bowel perforation increases with age, CTC in lieu of colonoscopy may be indicated in some older individuals.

\section{Lung cancer}

In a large randomized controlled study, yearly low dose CT of the lung reduced the risk of cancer-related $(20 \%)$ and overall mortality (6\%) in high risk individuals ages 55-75 [24]. Current smokers and ex-smokers who had quit the habit within 15 years were considered high risk. These results are particularly relevant to older individuals because lung cancer-related mortality increases with age [25] and mortality for lung cancer in the aged concerns mainly ex-smokers [26]. Lingering questions include the number of and the interval between screening sessions, the risk of exposure to low-dose radiation, and the benefits of screening beyond age 75 [27]. Until more data emerge, we feel that some form of screening should be offered to all individuals at risk with a life expectancy of 5 years or longer because lung cancer may be deadly in 5 years, and safer alternatives to open thoracotomy exist today for the treatment of early disease. These include minimally invasive surgery [28], stereotactic radio surgery [29], and radiofrequency and thermo-ablation [30].

\section{Prostate cancer}

The screening of asymptomatic men for prostate cancer with serial PSA determinations has been controversial for the last 20 years, based on the impression that prostate cancer is generally an indolent disease and is not life-threatening, that the diagnostic confirmation of prostate cancer involved multiple prostate biopsies that were both painful and risky, and that the primary treatment involved either radical prostatectomy or external beam radiation, both of which are associated with severe and permanent complications [31]. A recent randomized prospective study [32], showing that monitoring of localized prostate cancer was associated with the same 10-year survival as immediate treatment with surgery or $\mathrm{RT}$, reinforced this impression that screening for prostate cancer is unnecessary and may be contra-indicated. In the same study the risk of metastatic disease at 10 years was higher among men managed with monitoring. Thus, with longer follow-up, monitoring without immediate treatment may lead to a poorer survival. Once again this finding indicates the need to base screening programs on life expectancy rather than chronologic age. For this reason we feel that the current USPSTF guidelines recommending against screening men 70 and older for prostate cancer should be reconsidered [33].

Three large randomized controlled trials [34-36] compared screening with serial PSA determinations and no screening. The PLCO (Prostate Lung Colon and Ovary) study included men ages 55-74 undergoing yearly PSA determination for 6 years and yearly digital rectal examination by a health professional for 4 years. Screening did not improve disease-specific and overall survival 
Citation: Vallet-Regi M, Manzano M, Rodriguez-Mañas L, et al. (2017) Secondary Prevention of Cancer in the Older Individual. Trends Geriatr Healthc 1(1):14-22

[35]. However the majority of individuals in the control group had one or more assessment of serum PSA at some time in the course of the study based on the decision of their personal physicians, so the results of the study can hardly be taken as proof of screening ineffectiveness. The European trial [34] involved men ages 55-69, and screening consisted of determination of PSA every 4 years on average and reported a decline of prostate cancer-related mortality with screening but not of overall mortality. The investigators also demonstrated that 1,011 men would need to be screened and 37 cases of prostate cancer diagnosed to prevent one cancer death. Likewise the Goteborg trial [36] demonstrated that regular screening reduced the prostate cancer mortality rate by $50 \%$ in men ages 55-69, and 293 men would need to be screened and 12 prostate cancers treated to prevent one death. Again, screening had no detectable effect on overall survival. Based on this data, the Cochrane review [37] concluded that a decline in prostate cancer-related mortality may be obtained through screening in men with a life expectancy of 10-15 years and that the frequent and serious complications of biopsy and treatment may counteract the benefits of screening.

These conclusions should be mitigated by the following considerations of special interest in terms of aged men:

- The risk of prostate cancer-related deaths increases with age at diagnosis at least up to age 89 [38]. Given the aging of the population one may predict that the incidence and mortality of prostate cancer in older individuals will increase.

- Serial biopsies of prostate cancer in men undergoing active monitoring of prostate cancer demonstrated a progressive increase in the grade of the same prostate cancer [39] with the aging of the patient. Along the same lines, prostate cancers diagnosed after age 80 generally appear more aggressive than those diagnosed in younger men [40].

- Multi-parametric MRI is more specific than trans-rectal ultrasonography in identifying high-risk prostate lesions and may reduce the number of biopsiesand related risk-necessary for diagnosing prostate cancer [41].

- New forms of treatment of localized disease-including image-modulated radiation therapy, proton therapy, and cryosurgery-may be associated with minimal risk of complications [42].

- It may become possible to identify individuals at high risk of developing prostate cancer. In addition to family history and ethnicity, these factors may include midlife PSA levels [43]. If these data are confirmed, screening may be limited to a small number of individuals who are at increased risk for prostate cancer.

The case of prostate cancer is paradigmatic of how one must approach cancer screening in the older person as a dynamic issue, whose variables include new and safer diagnostic and therapeutic interventions in addition to the aging of the population.

\section{Hepatocellular carcinoma}

The mortality of hepatocellular carcinoma may be reduced by screening individuals affected by hepatitis $\mathrm{B}$ and $\mathrm{C}$ with serial liver ultrasound and alpha fetoprotein determinations [44]. Any person at risk with a life expectancy of 5 years or longer may benefit from this intervention. This recommendation should also include individuals unable to tolerate a hepatectomy, as alternative forms of treatment $[45,46]$, such as thermo-ablation or hepatic artery chemo and radio-embolization, or stereotactic radio surgery, are tolerable even by frail patients.

\section{Cervical cancer}

There has been a progressive increase in the incidence and mortality of cervical cancer in older women [47]. The cause of this phenomenon is unknown and may be in part related to the prolongation of the sexual life of the aged. Current screening recommendations for cervical cancer are based on a large epidemiologic study showing that serial PAP smear examination of the cervix had led to a $75 \%$ decline in mortality of younger women $[13,47]$. The recommendation to stop screening at age 60 should be revisited in view of this spate of cervical cancers in later ages [47].

\section{Screening Older Individuals for Cancer: A Dynamic Approach}

As outlined in the previous review, four important factors influence the discussion of screening older individuals with cancer. These include the aging of the population, new insights in cancer biology, new insights in the assessment of aging that allows the practitioner to estimate individual physiologic age [48-54], and the development of safer and more specific diagnostic and treatment interventions. The combination of these elements represents a twofold challenge: how to collect new evidence and how to apply current evidence to clinical decisions.

\section{How to collect new evidence}

Large randomized clinical trials may have become obsolete in the new reality. While we are still discussing the benefit of mammographic screening for breast cancer, new imaging techniques may make mammography outdated. In addition, due to strict selection criteria, randomized controlled trials cannot encompass the diver- 
Citation: Vallet-Regi M, Manzano M, Rodriguez-Mañas L, et al. (2017) Secondary Prevention of Cancer in the Older Individual. Trends Geriatr Healthc 1(1):14-22

sity of the older population in terms of life expectancy, tolerance of stress, functional independence and social support [55]. These elements determine one's physiologic age and may determine the benefits and risks of secondary cancer prevention more than do chronologic age.

Still, long-term follow ups of past trials may provide important age-related information. For example, prostate cancer screening trials may establish whether the screening of men in their late 60 s results in a reduction of cancer-related mortality 20 years later, as early treatment of cancer may have prevented the development of a more aggressive cancer late in life. Likewise, age-related recommendations based on many of those trials may have become obsolete given the progressive increase inhuman life expectancy in addition to new biological insights and clinical developments.

As long as patients are selected on the basis of physiologic age [52-55], randomized controlled trials still have an important role in addressing age-specific specific questions such as:

- Do more sensitive breast-imaging techniques than mammograms reduce the risk of breast cancer related mortality in women 75 (or 80 ) and older with a life expectancy of 5 years?

- Does the use of FIT or fecal DNA decrease the useand the risks-of colonoscopy in persons 70 and older with a life expectancy of at least 5 years?

- Do biological markers of aging [48-51] predict mortality risks better than clinical evaluation in patients undergoing screening?

The SEER data on mammography have demonstrated the usefulness of registry databases in providing information on long-term mortality risk and survival of older individuals following cancer screening. Thanks to electronic records, tumor registries may be developed into rapid-learning databases [56]. These new instruments, exemplified by the ASCO cancer LinQ [56], may be ideal to collect and interpret information on the values of screening asymptomatic older individuals for cancer. Unlike traditional registries, rapid-learning databases may encompass the diversity of the older population and allow the estimate of the physiological age of each individual. In addition, they have the flexibility to accommodate new technological and biological information and the capability to analyze new information in a timely and efficient manner $[55,56]$. All

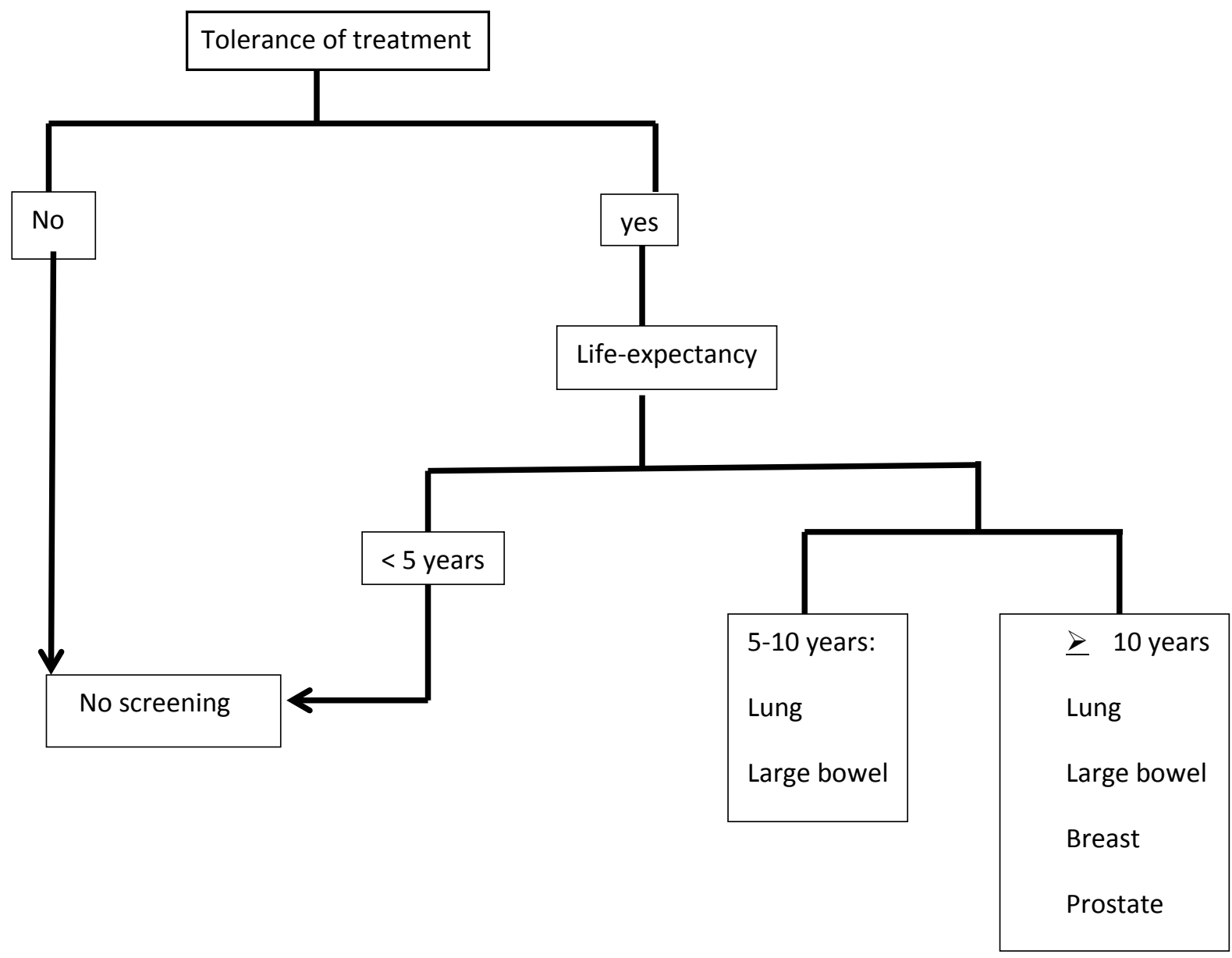

Figure 1: Decision tree to guide the screening of older individuals for breast cancer. 
patients included in the database should undergo the same basic clinical evaluation, including life expectancy and suitability for treatment (Figure 1).

\section{Practical approach to screening older individuals for cancer based on current evidence}

In Figure 1 we propose a decisional tree to establish which older individuals may benefit from cancer screening. The ability to tolerate treatment will be an issue only rarely. We have already illustrated how local treatment of lung, prostate and breast cancer may be minimally invasive and well tolerated even by individuals with limited functional reserve.

The determination of physiologic age is critical to maximize the benefits and minimize the risk of screening (Table 4). Currently, the comprehensive geriatric assessment (CGA) has been utilized for this purpose [56]. Based on the CGA, one may estimate the risk of cancer-unrelated mortality up to 9 years [52] and the risk of complications from some anti neoplastic treatment such as surgery and cytotoxic chemotherapy [53,57]. Of the tests of physical functions, the timed "get up and go" and the gait speed are probably the ones most widely used [58-59]. Both may predict the risk of death and the risk of functional dependence, but neither has been validated to predict the risk of complications from oncological treatments. There are at least two different constructs of frailty [60]: one includes a condition of increased risk of mortality, disability and institutionalization over a 10 year period; the other involves a condition of almost exhausted functional reserve for which minimal stress may precipitate functional dependence. The first concept has been operationalized in the now classic Fried's criteria [60] and has never been studied in cancer patients. The second construct was operationalized in four different instruments of functional assessment in which frailty was the lowest functional status [61]. In a prospective study comparing the accuracy of the four instruments, frailty was predictive of 1 year mortality in cancer patients 70 and over. Frailty in this situation identifies patients with very limited life expectancy and tolerance of stress. Seemingly these individuals represent only a small fringe of those who are not candidates for cancer screening.
Aging is associated with chronic and progressive inflammation, assessed with the inflammatory index; changes in DNA methylation, assessed with the epigenetic clock; and expression of anti proliferative genes, such as the expression of P16 INK4a in circulating lymphocytes. Each one of these biologic markers of aging predicts the risk of mortality and functional dependence in the general population of individuals 65 and older [48-50]. For reasons not well understood, aging is also associated with decreased concentrations of Vitamin D in the circulation, and Vitamin D levels are also an independent variable, a harbinger of mortality in the older population [51]. Seemingly, they may be utilized to finetune the assessment of physiologic age. Assessment of the Redox status and telomere length have also been proposed as markers of aging [10]. However, the Redox status does not add additional information to the clinical assessment, and the inter individual variability of the telomere length is too high to provide a reliable marker of aging.

\section{Conclusions}

Cancer in the older aged person is an increasingly common problem, and age is currently a risk factor for cancer-related mortality. This review demonstrates that secondary prevention (early diagnosis of cancer by screening asymptomatic individuals) may reduce cancer-related mortality in the older population.

The benefit of cancer screening may be lessened and the risk increased with age, due to an age-related decline in life expectancy and in tolerance of stress, which may include cancer treatment. Thus the decision to screen older individuals should be based on physiological rather than chronological age. Based on current information, it is reasonable to offer some form of screening to individuals with a life expectancy of 5 years or more for colorectal and lung cancer and to those with a life expectancy of 10 years or more for breast and prostate cancer. It is also reasonable to extend screening for cervical cancer to women above age 60 if they still have a uterus.

Unanswered questions include:

- The role of new imaging techniques for early detection of breast cancer and of TIS and fecal DNA for early detection of colorectal cancer

Table 4: Determination of physiologic age.

\section{Clinical determination}

Comprehensive Geriatric Assessment (CGA)

Tests of physical function

Frailty

Laboratory determination
Inflammatory status (inflammatory index)
Epigenetic clock (methylation status)
Concentration of P16 INK4a in circulating lymphocytes
Circulating levels of Vitamin D
Length of leukocyte telomere
Redox status


Citation: Vallet-Regi M, Manzano M, Rodriguez-Mañas L, et al. (2017) Secondary Prevention of Cancer in the Older Individual. Trends Geriatr Healthc 1(1):14-22

- The best approach to the screening of colorectal cancer (whether biannual stool examination, flexible sygmoidoscopy every 3-5 years, or colonoscopy every 10 years)

- The best number and the best interval among screening sessions for breast, prostate, and lung cancer

The main challenge in studying cancer screening in the future includes the diversity of the aging population, the progressive increase in life-expectancy, the development of more sensitive screening techniques, and new insights into the biology of cancer and aging. Rapid-learning databases rather than randomized clinical trials may represent the most reliable instruments to accommodate and interpret this mounting wealth of new information.

\section{Acknowledgment}

Maria Vallet-Regi thanks funding from the European Research Council (Advanced Grant VERDI; ERC-2015AdG Proposal no. 694160).

\section{References}

1. Shih YC, Hurria A (2014) Preparing for an epidemic: cancer care in an aging population. Am Soci Clin Oncol Educ book 2014: 133-137.

2. Van de Water W, Markopoulos C, va de Velde C, et al (2012) Association between Age at Dignosis and DiseaseSpecific Mortality among postmenopausal women with Hormone-receptor positive Breast cancer. JAMA 307: 590597.

3. Bairey O, Ruchlemer R, Rahimi-Levene N, et al. (2011) Presenting features and outcome of chronic lymphocytic leukemia in patients diagnosed at age of 80 and older. An ICLLSG study. Ann Hematol 90: 1123-1129.

4. SEER cancer statistics Review, 2009.

5. Soung MC (2015) Screening for cancer: when to stop?: A Practical guide and review of the evidence. Med Clin N America 99: 249-262.

6. Silvennoinen M, Mecklin JP, Saariuloma P, et al. (2009) Expertise and Skill in minimally Invasive Surgery. Scand J Surg 98: 209-213.

7. Torrens M, Chung C, Chung HT, et al. (2014) Standardization of terminology in stereotactic radiosurgery. Report from the standardization Committee of the International Leksell gamma knife society: special topics. J Neurosug 121: 2-15.

8. Millner LM, Strotman LN (2016) The future of Precision Medicine in Oncology. Clin Lab Med 36: 557-573.

9. Yourman LC, Lee SJ, Schonberg MA, et al. (2012) Prognostic indices for older adults: a systematic review. JAMA 307: 82-192.

10. Balducci L (2016) Cancer prevention in the Older Individual. Semin Oncol Nurs 32: 314-324.

11. Lee CP, Cherlow GM, Zenios SA (2009) An empiric estimate of the value of life: updating the renal dialysis cost-effectiveness standard. Value Health 12: 80-87.
12. Balducci L, Fossa SD (2013) Rehabilitation of Older cancer patients. Acta Oncol 52: 233-238.

13. Comparetto C, Borruto F (2015) Cervical cancer screening: A never-ending developing program. World $\mathrm{J}$ Clin Cases 16: 614-624.

14. Gøstche PC, Jørgensen KG (2013) Screening for Breast Cancer with Mammography. Cochran Database of systematic Reviews.

15. Nelson HD, Cantor A, Humphrey L, et al. (2016) Screening for Breast Cancer: a Systematic Review to Update the US Preventive Service Task Force Recommendation. Agency for Healthcare Research and Quality, Rockville.

16. Miller AB, Walls C, Baines CJ, et al. (2014) Twenty year follow up for breast cancer incidence and mortality of the Canadian National Breast Screening Study: randomized screening trial. BMJ 348: 366.

17. McPherson CP, Swenson KK, Lee MW (2002) MW the effects of mammographic detection and comorbidity on the survival of older women with breast cancer. J Am Ger Soc 50: 1061-1068.

18. Broedeers MJ, Verbeek AL, Straatman H, et al. (2002) Repeated mammographic screening reduces breast cancer mortality along the continuum of age. J Med Screen 9: 163167.

19. Lash TL, Fox MP, Buist DS, et al. (2007) Mammography surveillance and mortality in Older Breast cancer Survivors. J Clin Oncol 25: 3001-3006.

20. Vacek PM, Skelly JM (2015) Prospective study of the use and effects of screening mammography in women aged 70 and over. J Am Geriatr Soc 63: 1-7.

21. Braithwaite D, Walter LC, Izano M, et al. (2016) Benefits and harms of screening mammography by comorbidity and age : a qualitative synthesis of observational Studies and Decision analysis. J Gen Intern Med 31: 561-572.

22. Lin JS, Piper MA, Perdue LA, et al. (2016) Screening for Colorectal Cancer : A Systematic Review for the US Preventive Services Task Force. Rockville (MD): Agency for Healthcare Research and Quality (US), Rockville.

23. Shergill AK, Cooners EE, McQuaid KR, et al. (2015) Protective association of colonoscopy against proximal and distal colon cancer and patterns in interval cancer. Gastrointest Endosc 82: 529-537.

24. Aberle DR, Adams AM, Berg CD, et al. (2011) Reduced lung cancer mortality with low dose computed tomographic screening. N Engl J Med 365: 395-409.

25. Knoke JD, Shanks TG, Waughn JW, et al. (2004) Lung cancer mortality is related to age in addition to duration and intensity of cigarette smoking An analysis of CPS1 data. Cancer Epidemiol Biomarkers Prev 13: 949-957.

26. Peto $J$ (2011) That lung cancer incidence falls in ex-smokers: misconceptrion 2. Br J Cancer 104: 389.

27. Bach PB, Mirkin JN, Oliver TK, et al. (2012) Benefits and Harms of CT screening for lung cancer. A systematic review. JAM 307: 2418-2429.

28. Li M (2015) Time to change: big show for revolution in the eigth national forum for minimally invasive surgery in lung cancer. J Thorac Dis 7: E278-E282.

29. Dajac J, Bhattal G, Keller A, et al. (2016) Evaluation of 
Citation: Vallet-Regi M, Manzano M, Rodriguez-Mañas L, et al. (2017) Secondary Prevention of Cancer in the Older Individual. Trends Geriatr Healthc 1(1):14-22

overall survival following stereotactic radiosurgery (SRS) for non small cell lung cancer. Int J Radiat Oncol Biol Phys 96: E485.

30. de Baere T, Tselikas L, Catena V, et al. (2016) Percutaneous thermal ablation of primary lung cancer. Diagn Interv Imaging 97: 1019-1024.

31. Loeb S (2016) Evidence-based versus personalized prostate cancer screening: using baseline prostate specific antigen measurements to individualize screening. J Clin Oncol 34: 2684-2686.

32. Hamdy MD, Donovan JL, Lane JA, et al. (2016) Ten year outcome after monitoring, surgery or radiotherapy for localized prostate cancer, N Engl J Med 375: 1415-1424.

33. Moyer VA (2012) Screening for prostate cancer: United States Preventive Service Task force recommendation statement. Ann Intern Med 157: 120-134.

34. Schröder FH, Hugossen J, Roobol MJ, et al. (2012) Prostate cancer mortality at 11 year follow-up. N Engl J Med 366: 981-990.

35. Andriole GL, Crawford ED, Grubb RL, et al. (2009) Mortality results from a randomized prostate cancer screening trial. N Engl J Med 360: 1310-1319.

36. Hugosson J, Carlsson S, Aus G, et al. (2010) Mortality results from the Goteborg randomized population based prostatecancer screening trial. The Lancet Oncol 11: 725-732.

37. Illic D, Neuberger MM, Djulbegovic M, et al. (2013) Screening for prostate cancer. Cochrane Database Syst Rev.

38. MacKintosh FR, Sprenkle PC, Walter LC, et al. (2016) Age and prosteate specific antigen level prior to diagnosis predict the risk of death from prostate cancer. Front Oncol 6: 157

39. Porten SP, Whitson JM, Cowan JH, et al. (2011) Changes in prostate cancer grade on serial biopsy in men undergoing active surveillance. J Clin Oncol 29: 2795-2800.

40. Bechis SK, Carroll PR, Cooperberg MR (2011) Impact of Age at Diagnosis on Prostate Cancer treatment and survival. J Clin Oncol 29: 235-241.

41. Busetto GM, De berardinis E, Sciarra A, et al. (2013) Prostate cancer Gene 3 and multiparametric magnetic resonance can reduce unnecessary biopsies: decision curve analysis to evaluate predictive models. Urology 82: 13551360.

42. Yeo I, Nookala P, Gordon I, et al. (2015) Passive proton therapy vs IMRT planning study with focal boos for prostate cancer. Radiant Oncol 10: 213.

43. Preston MA, Batista JL, Wilson KM, et al. (2016) Baseline prostate-specific Antigen level in midlife predict lethal prostate cancer. J Clin Oncol 34: 2705-2711.

44. Wu CY, Hsu YC, Ho HJ, et al. (2016) Association between ultrasonographic screening and mortality in patients with hepatocellular carcinoma: a nationwide cohort study. Gut 65: 693-701.

45. Ryan MJ, Willatt J, Majdalany BS, et al. (2016) Ablation techniques for primary and metastatic liver tumors. World $\mathrm{J}$ Hepatol 8: 191-199.
46. Bhangoo MS, karnani DR, Hein PN, et al. (2015) Radioembolization with Yttrium 90 microspheres for patients with unresectable hepatocellular carcinoma. J Gatrointest Oncol 6: 469-478.

47. Rositch RF, Nowark RG, Gravitt PA (2014) Increased age and race-specific incidence of cervical cancer after correction of hysterectomy prevalence in the Unites States from 2000 through 2009. Cancer 120: 2032-2038.

48. Marioni RE, Shah S, McRae AF, et al. (2015) DNA methylation age of blood predicts all causes mortality in later life. Genome Biol 16: 25.

49. Varadhan R, Yao W, Matteini A, et al. (2014) Simple biologically informed inflammatory index of two serum cytokines predicts 10 year all cause mortality in older adults. J Gerontol A Biol Sci Med Sci 69: 165-173.

50. LaPak KM, Burd CE (2014) The Molecular balancing act of P16 (INK4a) in cancer and aging. Molec Cancer res 12: 167-183.

51. Schöttker B, Jorde R, Peasey A, et al. (2014) Vitamin D and mortality: a meta-analysis of individual participant data from a large consortium of Cohort studies from Europe and the United States. BMJ 348: 3656.

52. McClymont KM, Lee SJ, Schmberg MA, et al. (2014) Usefulness and effects of online prognostic calculators. J Am Geriatr Soc 62: 2444-2445.

53. Cohen HJ, Smith D, Sun CL, et al. (2016) Frailty as determined by a comprehensive geriatric assessment-derived deficit-accumulation index in older patients with cancer who receive chemotherapy. Cancer 15: 3865-3872.

54. Wildiers H, Heeren P, Puts $M$, et al. (2014) International Society of geriatric Oncology Consensus on Geriatric Assessment in Older Patients with Cancer. J Clin Oncol 32: 2595-2603.

55. Vallet-Regi M, Manzano M, Rodriguez-Mañas L, et al. (2017) Management of Cancer in the Older-Aged person: An approach to complex medical decisions. Oncologist 22: 335-342.

56. Shah A, stewart AK, Kolacevski A, et al. (2016) Building a rapid learning Health care system in oncology: why CancerLinq collects identifiable health Information to achieve its vision. J Clin Oncol 34: 756-763.

57. Audisio RA, Pope D, Ramesh HS, et al. (2008) Shall we operate? Preoperative Assessment in elderly cancer patients (PACE) can help. A SIOG surgical task force prospective study. Crit Rev Oncol Hematol 65: 156-163.

58. Tangalos EG (2015) get up and go. J Med Dir Ass 16: 638639.

59. Perera S, Patel KV, Rosano C, et al. (2016) Gait speed predicts incident disability: A Pooled Analysis. J Gerontol A Biol Sci Medical Sci 71: 63-71.

60. Balducci L (2013) Frailty: A common pathway in aging and cancer. Interdisc Top Gerontol 38: 61-72.

61. Ferrat E, Paillaud E, Caillet P, et al. (2017) Performance of four frailty classifications in Older Patients with Cancer: Pospective Elderly Cancer Patients Cohort Study. J Clin Oncol 35: 766-777. 